\title{
EDEMA CEREBRAL LOCALIZADO COMO COMPLICAÇÃO TARDIA DE TRAUMATISMO CRÂNIO-ENCEFÁLICO
}

\author{
Gilberto Machado de Almeida * \\ PedRo HenRIQUe Longo **
}

\begin{abstract}
O edema cerebral localizado secundário a traumatismos cranianos agudos não constitui novidade ${ }^{2,3}$. Entretanto, raras vêzes é lembrada esta eventualidade no diagnóstico diferencial das complicações tardias dos traumas crânio-encefálicos. De modo esquemático o quadro se caracteriza por: a) instalação tardia ( 30 a 60 dias após o traumatismo craniano) de sinais sugestivos de processo expansivo intracraniano; b) achados neuro-radiológicos demonstrando diferença de volume entre os hemisférios cerebrais; c) regressão da sintomatologia com tratamento medicamentoso.
\end{abstract}

\section{OBSERVAÇOES}

Caso 1 - J. R. D., sexo masculino, branco, brasileiro, com 37 anos de idade, atendido no Pronto Socorro em 25-3-1954 (Reg. Geral 360.480). Tratava-se de um etilista crônico que, 30 dias após traumatismo de crânio, entrou em estado de mal epiléptico com convulsões que se inicjavam no hemicorpo direito; quando cessavam as convulsões restavam hemiparesia direita e afasia. O craniograma não mostrou iraturas e o exame do liqüido cefalorraquidiano revelou apenas discreta hipercitose (20 células por $\mathrm{ml}$, linfomononucleares). Electrencefalograma: diminuição da atividade elétrica na região parietal esquerda. Carotidoangiografia esquerda: acentuado desvio global da artéria cerebral anterior para a direita (fig. 1, A). Pneumoventriculografia: desvio de todo o sistema ventricular para a direita.

Em virtude da impossibilidade de localizar precisamente o "processo expansivo" e tendo em conta a progressiva melhora clinica decidiu-se pela não intervençāo cirúrgica. Após 50 dias, durante os quais foi administrada apenas medicação visando combater o edema cerebral, a repetição da pneumografia e da carotidoangiografia mostrou apenas dilatação do sistema ventricular que não mais se apresentava desviado (fig. 1, B). Novo electrencefalograma mostrou reduçāo acentuada da assimetria assinalada no primeiro exame, embora ainda com desorganização difusa. Na revisão do caso em ambulatório, 80 dias após a internação, restavam, como sequielas, hemiparesia direita e disfasia.

Trabalho do Serviço de Neurologia da Fac. Med. da Univ. de São Paulo (Prof. Adherbal Tolosa), apresentado ao II Congresso da Sociedade Brasileira de Neuroclrurgia (Campos do Jordão, SP), em 11. setembro 1959. * Neurocirurgião do Pronto Socorro. ** Neurorradiologista. 

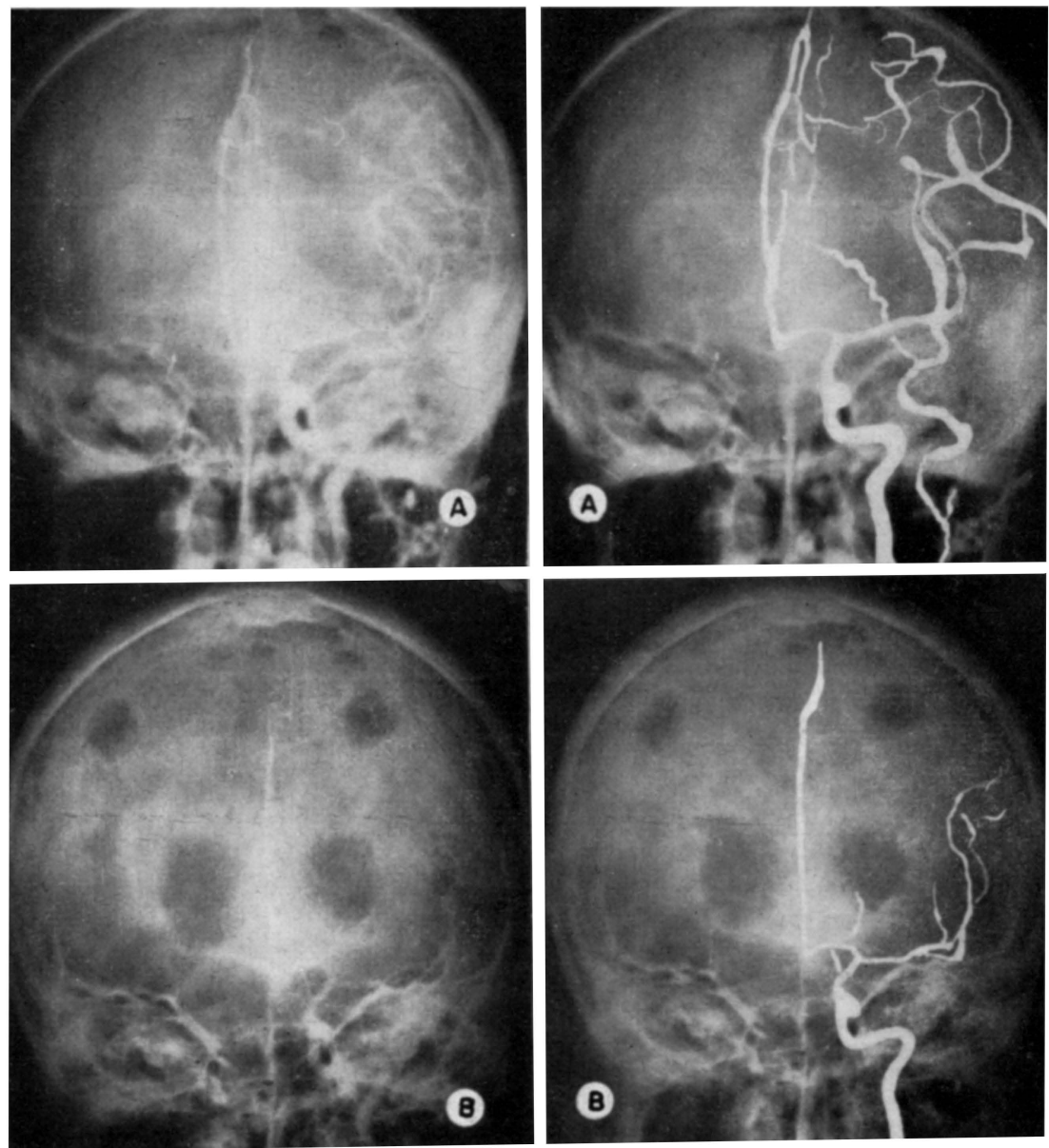

Fig. 1 - Caso 1 (J. R. D.). Em A, carotidoangiografia d̀ esquerda: desvio da artéria cerebral anterior para a direita. Em $B$, angiografia cerebral, realizada 50 dias após a primeira, mostrando a volta da artéria cerebral anterior para a linha média.

Caso 2 - A. M., sexo masculino, branco, brasileiro, com 14 anos, atendido no Pronto Socorro em 19-2-1957 (Reg. Geral 466.029). Tratava-se de paciente com ferimento corto-contuso e fratura linear na regiāo frontal direita que apresentou crise convulsiva generalizada no momento da internação. Submetido a tratamento médico, o paciente teve alta curado após 6 dias. Decorridos 2 meses, foi reinternado por apresentar sonolência, cefaléia e vômitos. O exame clínico-neurológico mostrou rigidez de nuca, papiledema bilateral e sinal de Babinski. Carotidoangiografia direita: quadro sugestivo de processo expansivo frontal direito (fig. 2,A). Visando esvaziamento de provável hematoma foi praticada uma perfuração frontal que revelou apenas edema cerebral. Foi instituida medicação anti-edema cerebral, obtendo-se, ao fim de 10 dias, regressão total do quadro clinico. O paciente foi mantido 
sob observaçāo em ambulatório durante os 19 meses seguintes, tendo nesse periodo apresentado duas crises convulsivas. Nova angiografia cerebral, feita no fim dêste período, foi inteiramente normal (fig. 2, B). Electrencefalograma mostrou anormalidade paroxistica frontotemporal direita.
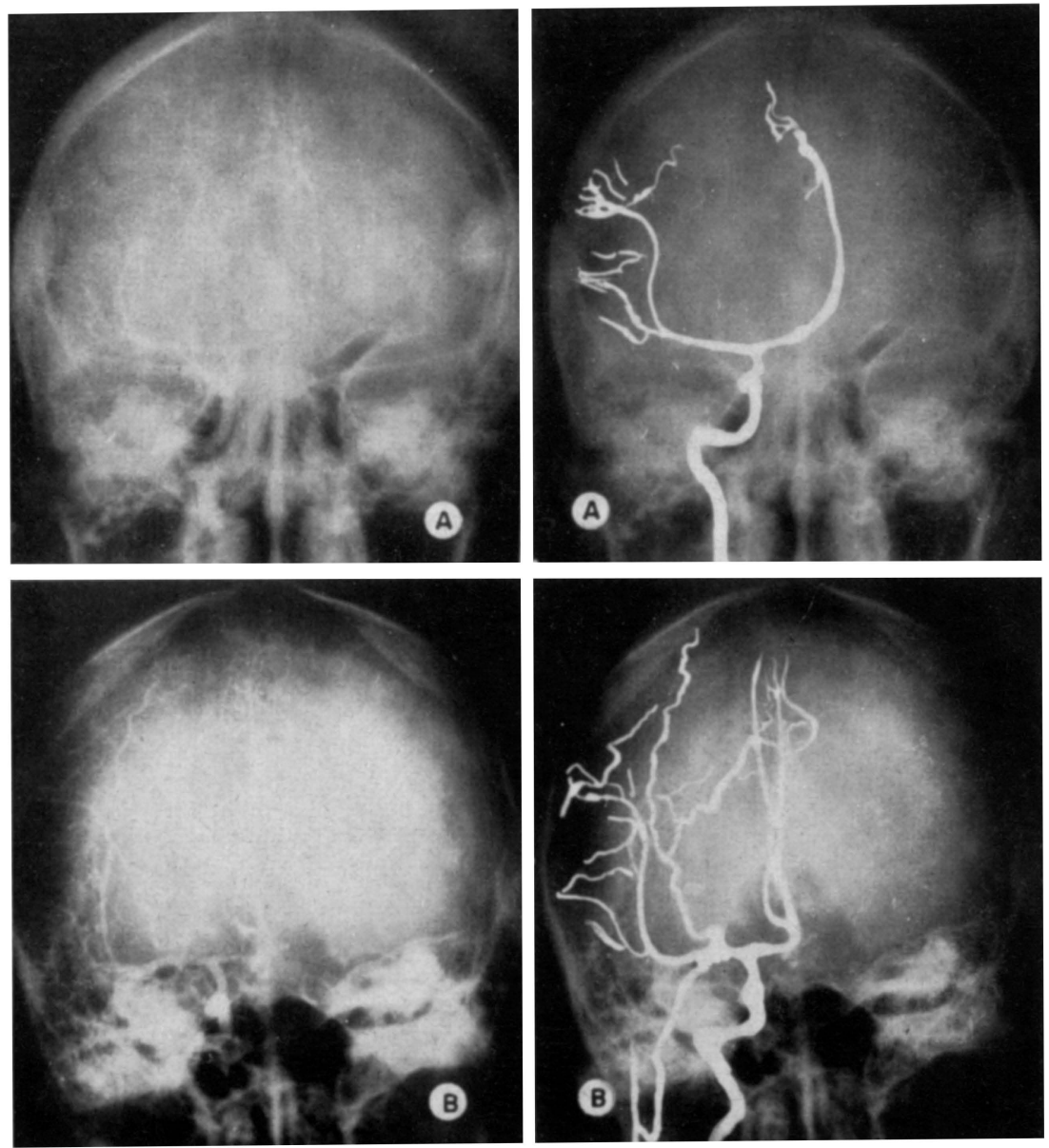

Fig. 2-Caso 2 (A. M.). Em A, angiografia cerebral à direita, mostrando desvio da artéria cerebral anterior para a esquerda, sugerindo processo expansivo frontal à direita. $\operatorname{Em} B$, repetiçáo do exame 19 meses após mostrando ausência do desvio observado no exame anterior.

Caso 3 - A. F. P., sexo masculino, branco, brasileiro, com 15 anos de idade, atendido no Pronto Socorro em 12-5-1957 (Reg. Geral 468.574). Paciente trazido ao hospital por apresentar cefaléia, febre e agitacão psicomotora há uma semana. Os familiares referiam traumatismo craniano 2 meses antes. Exame clínico-neurológico: hemiparesia flácida à esquerda, rigidez de nuca, papiledema bllateral, hiper- 
termia; após o exame ocorreu crise convulsiva atingindo $o$ hemicorpo esquerđo. Carotidoangiografia direita: desvio da artéria cerebral anterior para a esquerda; área suspeita de circulação anormal sōbre o plano mediano. Electrencefalograma: sinais de sofrimento cerebral em todo o hemisfério direito. Pan-angiografia cerebral, feita 10 dias após a internação: desvio das artérias cerebrais anteriores para a esquerda (fig. 3, A). Pneumoventriculografia sugerindo a existencia de processo expansivo frontotemporal direito. Com o tratamento medicamentoso que vinha sendo adminjstrado, houve melhora acentuada do quadro clínico (inclusive redução do papiledema), razāo pela qual foi adotada conduta expectante. Dois meses após a internação, nova pan-angiografia mostrou redução acentuada do desvio da artéría ce-
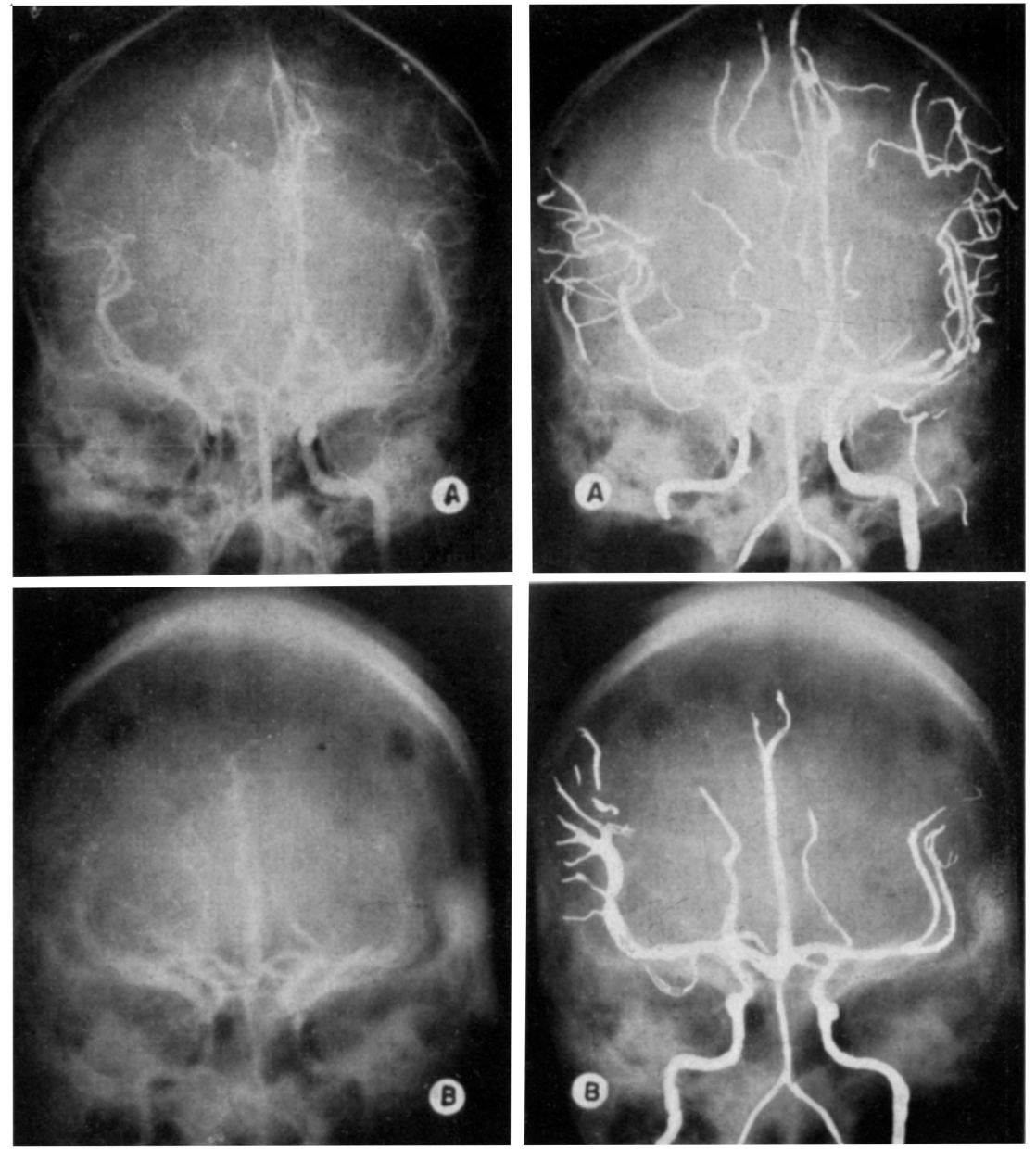

Fig. 3 - Caso 3 (A. F. P.). Em A, pan-angiografia cerebral mostrando desvio das artérias cerebrais anteriores para a esquerda. Em $B$, pan-angiografia feita 60 dias após a anterior mostrando as artérias cerebrais anteriores situadas no plano médio sagital. 
rebral; novo electrencefalograma revelou nitida melhora em relação ao exame anterior. Uma última pan-angiografia cerebral, felta às vésperas da alta hospitalar, mostrou aperas sinals indiretos de discreta dilatação ventricular (fig. 3, B). Na ocasião da alta ( 75 dias após a internação), o paciente apresentava, como seqüela, apenas discreto déficit motor no membro inferior esquerdo.

\section{COMENTARIOS}

Nos três casos os dados anamnésticos, clinicos e complementares levavam à suspeita da existência de hematoma subdural tardio pós-traumático. O electrencefalograma (casos 1 e 3 ), a arteriografia (casos 1,2 e 3 ) e a pneumoventriculografia (casos 1 e 3 ) mostraram apenas aumento de volume e sofrimento de um hemisfério cerebral, não permitindo maior precisão quanto ao diagnóstico topográfico. Entretanto, a evolução clínica, electrencefalográfica e radiológica demonstrou tratar-se de processo reversivel mediante o emprêgo de medicação visando o edema cerebral. A revisão do conjunto dos dados obtidos sugere que o aumento localizado do volume de um dos hemisférios cerebrais, tenha sido devido a edema encefálico tardio. No emprêgo da expressão "edema cerebral" não está implicada suposição alguma quanto ao tipo histológico do processo, mesmo porque não há elementos que nos permitam considerações a respeito. Encarando o problema sob um ponto de vista puramente clínico, o edema encefálico localizado tardio pode ser enquadrado entre os "pseudo-tumores", com assimetrias e deslocamentos ventriculares ${ }^{1}$.

Quanto às seqüelas, em um dos casos permaneceram crises de tipo epiléptico e nos dois outros, permaneceu discreto déficit motor. Electrencefalogramas e exames radiológicos feitos durante o seguimento dos casos confirmaram a existência de lesões cerebrais predominantemente do tipo atrófico. Esses dados permitem admitir que o edema localizado progressivo decorrente de pequena contusão cerebral seria o responsável pelo quadro subagudo que levou os pacientes ao Pronto Socorro e que as seqüelas teriam como causa as lesões iniciais agravadas por um edema transitório.

$\hat{E}$ interessante salientar que os três pacientes foram atendidos em um periodo de 4 anos; nesse mesmo período, no mesmo Serviço de Pronto Socorro foram diagnosticados e confirmados cirùrgicamente 91 casos de hematoma subdural, 111 de hematoma extradural e 28 de hematoma intracerebral. Talvez pela sua baixa incidência o edema cerebral localizado não teve o merecido destaque na apreciação das complicaçōes tardias dos traumatismos crânio-encefálicos. No entanto, pela diversidade das condutas a serem adotadas, o diagnóstico diferencial com os hematomas intracranianos (especialmente com o subdural) torna-se imperativo. Aparentemente isto não é possível apenas com os dados clínicos; mesmo o exame angiográfico, embora de grande valia, não é infalivel, pois a falta de área avascular não exclui a possível existência de hematoma extra ou subdural. Dêsse modo, o diagnóstico de edema cerebral localizado pós-traumático só será firmado após 
exploração cirúrgica, ou então, no caso de ser adotada conduta expectante, pela evolução- clinica, electrencefalográfica e arteriográfica.

\section{RESUMO}

Entre os pacientes internados, por traumatismos cranianos, no Pronto Socorro de Neurocirurgia do Hospital das Clínicas da Faculdade de Medicina de São Paulo, de 1954 a 1957, foram selecionados três, nos quais a evolução ulterior infirmou o diagnóstico inicial de hematoma extra ou subdural. O aparecimento, algum tempo após o traumatismo, de sinais focais e de hipertensão intracraniana sugeriu a existência de hematoma intracraniano provàvelmente subdural, sendo então indicada a realização, com urgência, de uma angiografia cerebral por via carotidea. Em todos os casos foram evidenciados desvios da artéria cerebral anterior, não havendo elementos para o diagnóstico da natureza do processo expansivo. Em dois casos foram feitas pneumografias que mostraram desvios do sistema ventricular e electrencefalogramas que mostraram depressão da atividade elétrica no hemisfério homolateral ao traumatismo. Exames radiológicos sucessivos mostraram diminuição progressiva, até a desaparição, dos desvios arteriais; um dos pacientes foi submetido a trepanaçāo exploradora que mostrou apenas edema cerebral. Medicação visando combater o edema cerebral determinou melhora acentuada dos quadros clínicos.

A finalidade do trabalho é de apresentar um quadro raramente descrito como complicação de traumatismos crânio-encefálicos e tecer comentários a respeito das dificuldades do diagnóstico diferencial com o hematoma subdural.

\section{SUMMARY}

Localized cerebral edema as late complication of cranio-cerebral trauma. Report of three cases.

A review of cases admitted to the "Emergency Service" of the Hospita] das Clínicas da Faculdade de Medicina da Universidade de São Paulo during a period of 4 years (1954 to 1957) was made and among them three cases were considered of interest because of the difficulty of diagnosis as well as its rarity. In those patients the evolution and further investigation invalidated the diagnosis of subdural hematoma with which they were admitted. This diagnosis had been made because of focal symptoms and intracranial hypertension after a period of one to two months after a craniocerebral trauma. Angiograms in the three cases had showed deviations of the anterior cerebral artery without sufficient data to demonstrate the nature of the expansive process. In two cases pneumoventriculographic examination showed a deviation of ventricular system and the electroencephalograms revealed depression of electric activity in the hemisphere ipsilateral to the traumatism. Successive roentgenologic examinations showed 
progressive reduction and even disappearing of the vascular deviation previously found. In one patient a trepanation was performed revealing nothing but cerebral edema. Conditions of the three patients were greatly improved with conservative measures. The object of this paper is to emphasize the difficulties for differential diagnosis with subdural hematoma.

\section{REFERENCIAS}

1. BARDECI, C. A.; PARVIS, D. A.; VAZQUEZ, E. - Seudotumores cerebrales con asimetria y desplazamentos ventriculares. Actas y trabajos del VI Congreso Latino-Americano de Neurocirurgia, págs. 1247-1255 (março) 1955. 2. LE BEAU, J.; FELD, M. - Traumatismes crânio-cérébraux fermés. Eucyclopédie Médico-Chirurgicale, Sec. Neurologie, vol. 3, fasc. 17.585, 1954. 3, PUECH, P. - Traumatismes Crânio-cérébraux. A. Legrand \& Cie., Paris, 1950.

Clínica Neurológica - Hospital das Clínicas da Fac. Med. da Univ. de São Paulo - Caixa Postal 3461 - São Paulo, Brasil. 